\title{
Fusion of 3D QCA and IVUS/OCT
}

\author{
Shengxian Tu • Niels R. Holm • \\ Gerhard Koning $\cdot$ Zheng Huang • \\ Johan H. C. Reiber
}

Received: 12 January 2011 / Accepted: 13 January 2011 / Published online: 25 January 2011

(C) The Author(s) 2011. This article is published with open access at Springerlink.com

\begin{abstract}
The combination/fusion of quantitative coronary angiography (QCA) and intravascular ultrasound (IVUS)/optical coherence tomography (OCT) depends to a great extend on the co-registration of $\mathrm{X}$-ray angiography (XA) and IVUS/OCT. In this work a new and robust three-dimensional (3D) segmentation and registration approach is presented and validated. The approach starts with standard QCA of the vessel of interest in the two angiographic views (either biplane or two monoplane views). Next, the vessel of interest is reconstructed in 3D and registered with the corresponding IVUS/OCT pullback series by a distance mapping algorithm. The accuracy of the registration was retrospectively evaluated on 12 silicone phantoms with coronary stents implanted, and on 24 patients who underwent
\end{abstract}

S. Tu $(\bowtie) \cdot$ G. Koning · J. H. C. Reiber

Division of Image Processing, Department of Radiology, Leiden University Medical Center, Albinusdreef 2,

2300 RC Leiden, The Netherlands

e-mail: S.T.Tu@lumc.nl

\section{N. R. Holm}

Department of Cardiology, Aarhus University Hospital, Skejby, Denmark

\section{Z. Huang}

Department of Cardiology, Nanfang Hospital Affiliated to Southern Medical University, Guangzhou, China

S. Tu · G. Koning · J. H. C. Reiber

Department of Applied Research, Medis Medical Imaging

Systems BV, Leiden, The Netherlands both coronary angiography and IVUS examinations of the left anterior descending artery. Stent borders or sidebranches were used as markers for the validation. While the most proximal marker was set as the baseline position for the distance mapping algorithm, the subsequent markers were used to evaluate the registration error. The correlation between the registration error and the distance from the evaluated marker to the baseline position was analyzed. The XA-IVUS registration error for the 12 phantoms was $0.03 \pm 0.32 \mathrm{~mm} \quad(P=0.75)$. One OCT pullback series was excluded from the phantom study, since it did not cover the distal stent border. The XA-OCT registration error for the remaining 11 phantoms was $0.05 \pm 0.25 \mathrm{~mm}(P=0.49)$. For the in vivo validation, two patients were excluded due to insufficient image quality for the analysis. In total 78 sidebranches were identified from the remaining 22 patients and the registration error was evaluated on 56 markers. The registration error was $0.03 \pm$ $0.45 \mathrm{~mm}(P=0.67)$. The error was not correlated to the distance between the evaluated marker and the baseline position $(P=0.73)$. In conclusion, the new XA-IVUS/OCT co-registration approach is a straightforward and reliable solution to combine X-ray angiography and IVUS/OCT imaging for the assessment of the extent of coronary artery disease. It provides the interventional cardiologist with detailed information about vessel size and plaque size at every position along the vessel of interest, making this a suitable tool during the actual intervention. 
Keywords Intravascular ultrasound · Optical coherence tomography · Quantitative coronary angiography · Three-dimensional reconstruction

\section{Introduction}

Over the past decades, the continuous developments in coronary visualization and quantitative systems have been motivated by the increasing need to better understand and assess coronary atherosclerosis and by the on-line need for support of coronary interventions in cardiac catheterization laboratories. Recently developed three-dimensional quantitative coronary angiography (3D QCA) systems aimed to resolve some of the limitations in conventional two-dimensional (2D) analysis [1-3] and hence, to extend its capacity and reliability in assessing the true dimensions of coronary vascular structures. It has been demonstrated that 3D QCA can accurately assess vessel segment length and diameter [4-7], as well as the optimal viewing angles [8-10] for the subsequent interventional stent-procedure. By using 3D QCA and based on such more accurate 3D data, clinical decision making can be affected, thus possibly leading to a more efficient and economic usage of stents in percutaneous coronary intervention (PCI) [11]. This may have significant impact in today's cost-constrained health care systems.

Despite the fact that the 3D angiographic reconstruction has important potential values, the foremost limitation of X-ray angiography-based systems remains the inability to image beyond the vessel lumen as only the contrast lumen is visualized. In other words, the 3D reconstructed vessel remains a lumenogram, though with better 3D capabilities. Thus, early stages of plaque formation may not be evident with X-ray angiography due to the occurrence of coronary artery remodeling [12], and vulnerable plaques can not be recognized for possible implementations of measures to prevent these from rupturing. These limitations have been well addressed by intravascular tomography-based imaging techniques, among which grey scale intravascular ultrasound (IVUS) is a well-established and validated modality. IVUS provides a wealth of information including vessel wall composition, which is crucial to the assessment of coronary atherosclerosis. Later on, the role of intravascular tomography-based imaging techniques was greatly enhanced by the radiofrequency data analysis for plaque characterization and optical coherence tomography (OCT) for the assessment of the thin fibrous cap atheromas and malapposition of stent struts. These new imaging techniques have extended the capabilities in the assessment of coronary artery disease. However, the fact that intravascular tomography-based imaging does not preserve the global topology information could lead to erroneous interpretations. Although a longitudinal view (L-View) is available in most IVUS/OCT consoles to provide an overview of the pullback series, the presentation of the L-View by stacking cross-sectional images along a straightened version of the transducer pullback trajectory is a very unnatural way of conceptualization. As a result, the interpretation can be quite challenging.

Given the different but complementary perspectives provided by X-ray angiography (XA) and IVUS/ OCT, the fusion/integration of the two imaging modalities by using XA as a roadmap while exploiting detailed vessel wall information from IVUS/OCT will benefit the interpretation of coronary artery disease and the guidance of coronary interventions. Currently, if IVUS/OCT is performed in the pre-intervention stage, the treatment planning is determined to a great extent by the IVUS/OCT interpretation. However, since XA fluoroscopy is still the only imaging tool available during stent deployment and positioning; the interventionalist must mentally establish the correspondence between XA and IVUS/OCT images. This spatial corresponding process is not always easy, especially for diseases of early stages, or long diffused lesions where lumen narrowing is not clearly evident and no sidebranch is present in the neighborhood of the lesion borders. Thus, XA-IVUS/OCT integrated systems are currently requested in the market to better support coronary interventions. The clinical applicability of such fused/integrated systems depends to a great extend on the reliability and robustness of the co-registration approach. Once a reliable correspondence between angiographic and IVUS/OCT images is established, the issue of fusing/integrating information from the two image modalities becomes relevant. 
In the following paragraphs our approach for the 3D reconstruction and co-registration with IVUS/ OCT is described and the results of both phantom and in vivo validations are presented.

\section{Three-dimensional angiographic reconstruction}

Accurate and robust $3 \mathrm{D}$ angiographic reconstruction is the foremost important step in the XA-IVUS/OCT coregistration. Early research on $3 \mathrm{D}$ reconstruction can be traced back to decades ago [13, 14]. However, clinic-ready systems were announced only in recent years and there have not been widespread acceptance of such systems in routine clinical practice. One of the reasons is due to the fact that mechanical distortions in $\mathrm{X}$-ray systems and noisy angiographic images in routine clinical acquisitions could significantly affect the reliability and robustness of the 3D reconstruction and analysis. For monoplane $\mathrm{X}$-ray angiographic acquisitions, the shift of the whole coronary tree due to the patient's respiration or the non-isocentric condition could greatly deteriorate the system's reliability. Such system distortions should be corrected before or during the 3D angiographic reconstruction.

A number of approaches [13-15] have been proposed to correct for angiographic system distortions. Ideally, a couple of reliable features, e.g., catheter tip and sidebranches, should be identified on the two angiographic views as reference points for the correction of system distortions. However, the practical applicability of such approaches in on-line usage has been hampered by the efforts in identifying many reliable features, which turned out to be too time consuming or even impossible to find such reliable features on the two angiographic views, especially when there were many overlaps from different vessel segments. To guarantee the reliability in the identification of reference points has already been a non-trivial task.

To come up with a more practical and attractive workflow, we have developed a new approach by using only one to three pairs of reference points for the correction of system distortions. In case of the presence of small perspective projection angles for noisy angiographic images, the reliability and robustness of the angiographic reconstruction are further improved by constructing a distance transformation matrix and by searching for the optimal corresponding path in the matrix to refine the correspondence between the two angiographic views [8]. The approach has been validated with high accuracy in both phantom and in vivo data [5, 8]. In short, the 3D angiographic reconstruction consists of only a few major steps: (1) load two image sequences acquired at two arbitrary angiographic views at least 25 degrees apart in viewing angles; (2) select the enddiastolic image frames with the vessel lumen well filled with contrast from the two image sequences for the subsequent 3D reconstruction; (3) identify one to three reference points in both angiographic views for the automated correction of system distortions; (4) manually define the vessel segment of interest and extract its contours and centerlines using our extensively validated QCA algorithms [1, 16, 17] in the two angiographic views; (5) reconstruct the 3D centerline and cross-sections after refining the correspondence between the two extracted centerlines.

An example of system distortions in the image geometry for the 3D angiographic reconstruction is given in Fig. 1a and b. The two bifurcation points (carina) in the left anterior descending artery (LAD) were identified as reference points and their epipolar lines, being the projection of the X-ray beam directed towards a particular point on one of the projections onto the second projection [13], were presented in the two angiographic views (31 RAO, 33 Cranial and 31 LAO, 30 Cranial, respectively). Due to the system distortions, the epipolar lines did not go through their corresponding reference points. After applying the automated correction of the system distortions, as shown in Fig. 1c and d, the epipolar lines now go right through their corresponding reference points in both angiographic views, demonstrating the success of this automated procedure.

Figure $2 \mathrm{a}$ and $\mathrm{b}$ show the segment of interest in the LAD and its extracted 2D contours, superimposed on the two angiographic views. Figure $2 \mathrm{c}$ shows the 3D reconstructed segment of interest under the viewing angle of 29 RAO, 9 Cranial. The subsegment (defined by the two superimposed markers) in the reconstructed vessel has a length of $16.10 \mathrm{~mm}$, per cent diameter obstruction of $38.0 \%$, and per cent area obstruction of $56.7 \%$. 


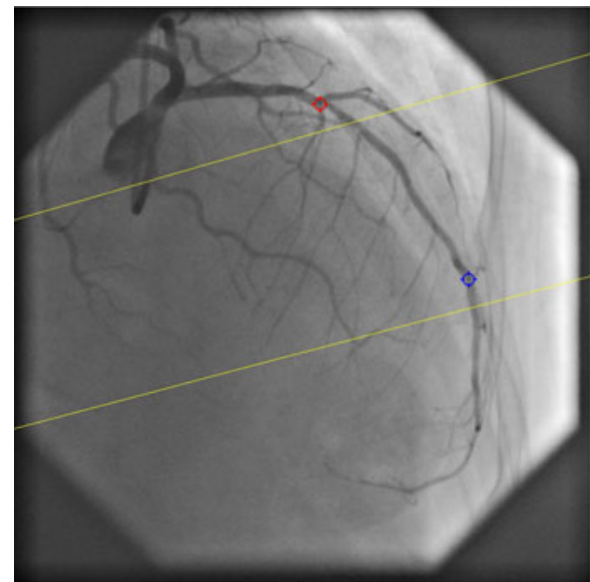

(a)

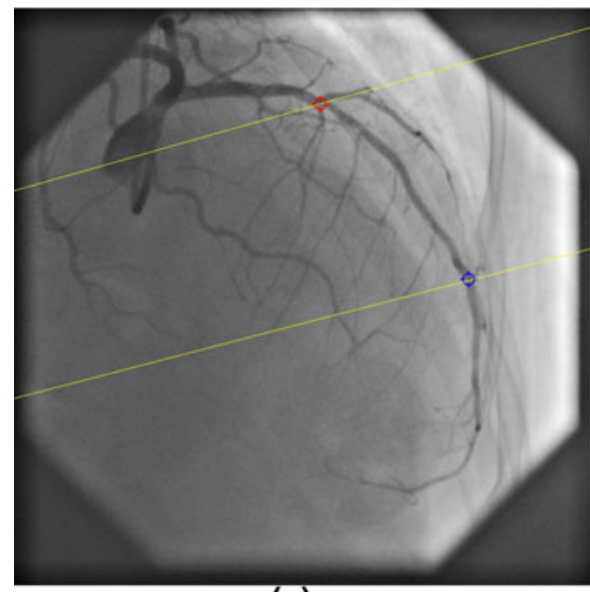

(c)

Fig. 1 Automated correction of system distortions in the image geometry for the $3 \mathrm{D}$ angiographic reconstruction: a and b are the two angiographic views (31 RAO, 33 Cranial and 31 LAO, 30 Cranial) selected for the 3D reconstruction. Before the correction, the two epipolar lines did not go through their

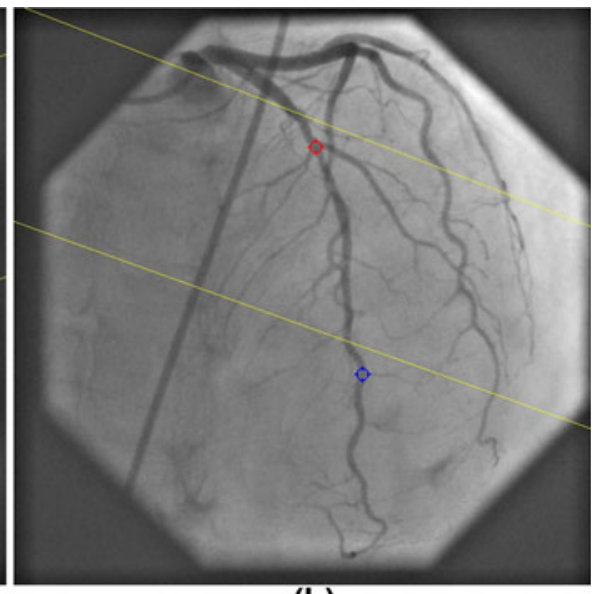

(b)

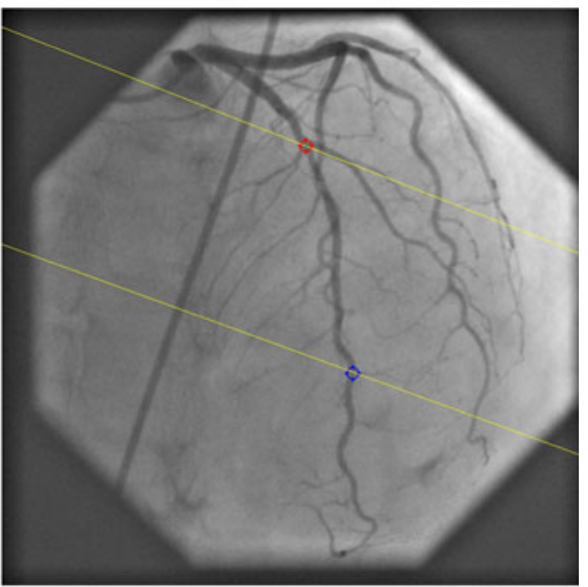

(d)

corresponding reference points, i.e., the two identified landmarks. $\mathbf{c}$ and $\mathbf{d}$ show the results after the automated correction of the system distortion. The two epipolar lines now go right through their corresponding reference points in both projection views

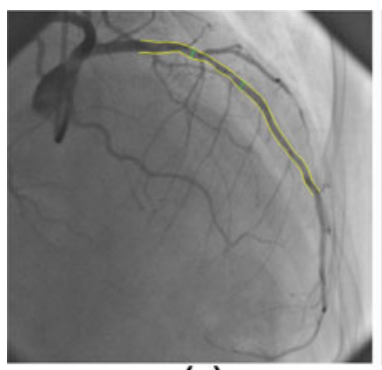

(a)

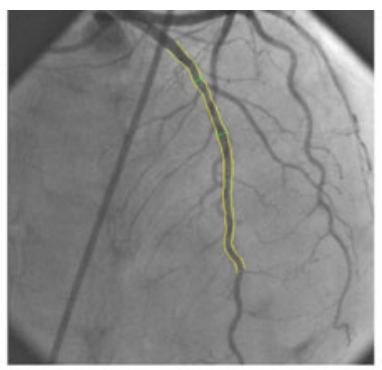

(b)

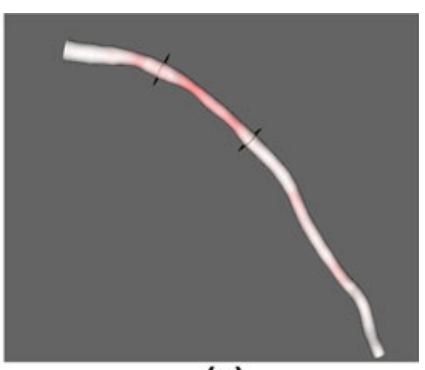

(c)

Fig. 2 The extracted 2D contours and the 3D reconstructed vessel segment of interest: $\mathbf{a}$ and $\mathbf{b}$ are the two angiographic views with the superimposed 2D contours; $\mathbf{c}$ is the $3 \mathrm{D}$ reconstructed vessel segment under 29 RAO, 9 Cranial 


\section{XA-IVUS/OCT registration}

Under the condition that the motorized transducer pullback with constant speed is used in the IVUS/OCT image acquisition, the rationale for the co-registration of XA images with IVUS/OCT pullback series is to use the spatial relationship between vessel segment (by means of lumen or centerline) and IVUS/OCT pullback trajectory. Conventional registration approaches [18-20] would require the reconstruction of the IVUS/ OCT imaging wire or sheath from two angiographic views acquired, and assume it to be the pullback trajectory so that the IVUS/OCT cross-sectional images can be aligned along the trajectory. This is not a trivial task due to the difficulty in segmenting both IVUS/OCT imaging wire and vessel lumen and the requirement of a second angiographic view for the IVUS/OCT catheter, which is not always included in the current workflow. The assumption of IVUS/OCT transducer path as pullback trajectory could also be jeopardized by the fact that spatial displacement of the imaging wire could occur inside the vessel after the pullback machine is switched on, in order to reach the state of minimum bending energy. It has been reported that there was significant delay from the moment the IVUS pullback machine was switched on and the moment the transducer tip really started to move [20].
In order to have a rapid and straightforward solution for the on-line XA-IVUS/OCT registration that could assist coronary interventions and would fit most into the current workflow in catheterization laboratories, we have taken a different approach by estimating the axial position of each IVUS/OCT cross-sectional image from the reconstructed vessel centerline, based on the curvature information and hence, to skip the reconstruction of the pullback trajectory. The approach only requires the operator to reconstruct the vessel centerline from the angiographic images (which is a standard module in 3D QCA software packages) and register it with IVUS/ OCT pullback series by indicating a baseline position in the vessel centerline that corresponds to the same axial position in IVUS/OCT. Such baseline positions can be found in anatomical or mechanical landmarks visualized in both angiographic and IVUS/OCT images, e.g., sidebranches and stent borders. In case of blurred angiographic images, image enhancement techniques [21] can be used to increase the visibility of detailed image structures.

After the registration, the markers superimposed on the angiographic views and the IVUS/OCT L-View are now synchronized. The interpretation of vessel dimensions becomes more comprehensive and the interventionalist now knows exactly where in the
Fig. 3 The XA-OCT co-registration and quantification. A sidebranch was manually identified from both XA and OCT images to be used as baseline position to register the two imaging modalities. After the registration, the markers superimposed in the OCT longitudinal view were synchronized with the same markers in the XA views. The OCT measurements could be compared with 3D QCA at the same position

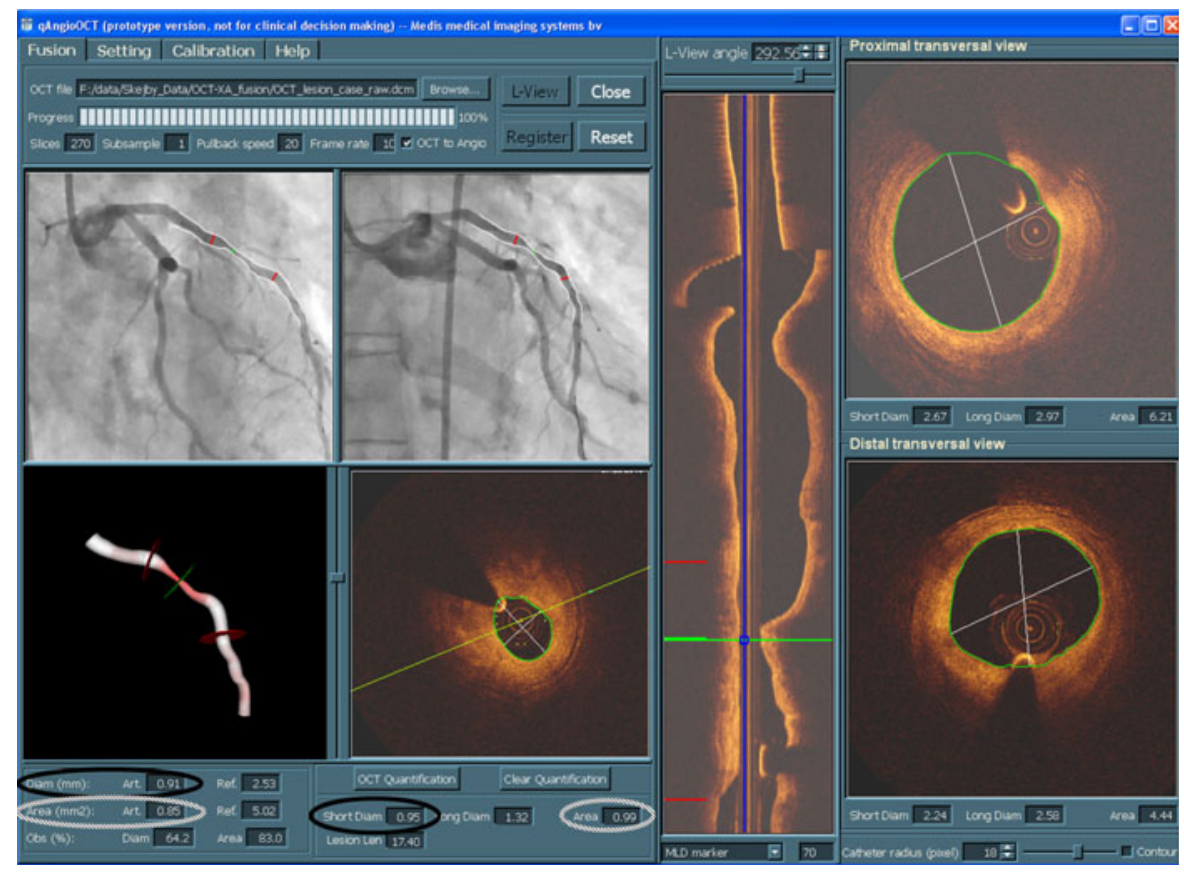


XA images the stent should be positioned. An example of combing QCA and OCT imaging is given by Fig. 3. After the registration, the planned stent position defined by the proximal and distal markers has been mapped onto the angiographic views (the two red markers that were superimposed on the angiographic views). In addition, the luminal contours were automatically detected in the OCT images by using a new minimum cost algorithm and the lumen diameter and area can be calculated and compared with the measurements from 3D QCA. In this example, the target vessel in the LAD has a minimum luminal diameter and area of $0.91 \mathrm{~mm}$ and $0.85 \mathrm{~mm}^{2}$ from 3D QCA, while the OCT measurements at the same position are $0.95 \mathrm{~mm}$ and $0.99 \mathrm{~mm}^{2}$, respectively.

\section{Validations}

Materials and methods

\section{Phantoms}

The accuracy of the registration was evaluated by acquiring a series of 12 different silicone phantoms (Via Biomedical, CA, USA) with coronary stents (Cypher Select+, Cordis, Johnson \& Johnson, Miami Lakes, Fla., USA) placed by the culotte two-stent technique. Main branch intracoronary acquisitions were used for the registration with the $3 \mathrm{D}$ angiographic reconstruction. Stent borders were used as markers for the validation. While the most proximal border was set as the baseline position for the distance mapping, the distal border was used to evaluate the registration error. The registration error was defined by the following protocol: Move a marker that was superimposed in the IVUS/OCT L-View to the position to be evaluated (in this case, the distal stent border); Move a second marker that was superimposed in the IVUS/OCT L-View to the position that corresponds to the same position to be evaluated in the angiographic views; The signed distance from the first to the second marker in the L-View was defined as the registration error.

For each phantom, the angiographic acquisitions were performed at two projections 60 degrees apart by a monoplane X-ray system (AXIOM-Artis, Siemens, Germany). The phantoms were filled with iodinated contrast media (Visipaque 270, GE Healthcare, WI, USA) during the acquisitions. Angiographic images were recorded at 25 frames/sec at a resolution of $512 \times 512$ pixels. To obtain IVUS images, the phantoms were immersed in water and acquisitions were performed at a constant pullback speed of $0.5 \mathrm{~mm} / \mathrm{s}$ by using a $20 \mathrm{MHz}$ transducer with a dedicated workstation (EagleEye Gold and s5, Volcano Corporation, Rancho Cordova, CA, USA) for six phantoms and a $40 \mathrm{MHz}$ transducer with a dedicated workstation (Atlantis SR Pro and iLab, Boston Scientific, Boston, MA, USA) for the other 6 phantoms. IVUS images were recorded at 30 frames/ sec and converted to DICOM format at a resolution of $512 \times 512$ pixels. To obtain OCT images, Fourier domain-OCT pullbacks were performed at $20 \mathrm{~mm} /$ sec by non-occlusive flushing technique using Visipaque 270 iodinated contrast media, and an OCT imaging catheter with a dedicated workstation (C7 Dragonfly and C7-XR, Lightlab Imaging, Westford, MA, USA). OCT images were recorded at 100 frames/sec and converted to DICOM format at a resolution of $1,024 \times 1,024$ pixels. Z-offset calibration was performed before converting to DICOM format for the subsequent analysis.

In vivo

At the Department of Cardiology, Nanfang Hospital affiliated to the Southern Medical University in Guangzhou, China, 24 patients who underwent both angiographic and IVUS examinations of the left anterior descending artery (LAD) were retrospectively selected for the validation. Inclusion criteria were: (1) patients had no prior history of coronary artery bypass surgery; (2) motorized pullback was used during the IVUS image acquisition; (3) angiographic images were recorded by digital flat-panel $\mathrm{X}$-ray acquisition systems.

Angiographic images were recorded at 25 frames/ sec by a monoplane X-ray angiogram (AXIOM-Artis, Siemens, Germany) at a resolution of $512 \times 512$ pixels. IVUS pullbacks were performed by using a motorized transducer pullback system $(0.5 \mathrm{~mm} / \mathrm{s})$ with a rotating $40 \mathrm{MHz}$ transducer catheter and $2.6 \mathrm{~F}$ imaging sheath (Boston Scientific, Boston, MA, USA). The sheath prevents direct contact of the imaging core with the vessel wall and increases stability of the pullback procedure. All parameters 
required by the $3 \mathrm{D}$ angiographic reconstruction and the co-registration were stored in DICOM files.

Reliable anatomical landmarks in the LAD, e.g., ostia of diagonal or septal branches, were identified from both angiographic and IVUS images and used as reference markers for the validation study. When IVUS pullbacks covered the left main bifurcation and the ostium of the left circumflex artery (LCX) was well visualized in angiographic images (no significant overlap with the proximal LAD), the left main bifurcation point (carina) was included as a reference marker. The LAD (including the left main if applicable) was reconstructed from two angiographic views and registered with IVUS pullback series by the distance mapping algorithm. While the most proximal reference marker was set as the baseline position for the distance mapping, the subsequent markers were used to evaluate the registration error. The registration error was defined using the same protocol as used in the phantom validation. The correlation between the registration error and the distance from the evaluated marker to the baseline position was analyzed.

\section{Statistics}

Quantitative data are presented as mean \pm standard deviation and the correlations were assessed by using Pearson's correlation coefficient. A 2 -sided $P$-value of $<0.05$ was considered to be significant. All statistical analyses were carried out by using a statistical software package (SPSS, version 16.0; SPSS Inc; Chicago, IL, USA).

\section{Results}

\section{Phantoms}

The lengths of the 12 stents in the main branches ranged from 12.00 to $32.00 \mathrm{~mm}$, with an average value of $22.92 \pm 7.26 \mathrm{~mm}$. The XA-IVUS registration error for the 12 IVUS pullbacks ranged from -0.33 to $0.57 \mathrm{~mm}$, with an average value of $0.03 \pm 0.32 \mathrm{~mm}$ $(P=0.75)$. For the OCT data, one pullback series was excluded from the study, since the pullback did not cover the distal stent border. The XA-OCT registration error for the remaining 11 OCT pullbacks ranged from -0.20 to $0.40 \mathrm{~mm}$, with an average value of $0.05 \pm 0.25 \mathrm{~mm}(P=0.49)$.
Table 1 Baseline characteristics

\begin{tabular}{ll}
\hline Age (years) & $60.5 \pm 13.2$ \\
Gender (male, female) & $17 / 5$ \\
1-vessel disease & $13(59 \%)$ \\
2-vessel disease & $7(32 \%)$ \\
3-vessel disease & $2(9 \%)$ \\
Target coronary artery $(n)$ & \\
Left anterior descending & 23 \\
Diagonal branches & 2 \\
Left circumflex & 5 \\
Right coronary artery & 3 \\
Location $(n)$ & \\
Proximal & 12 \\
Mid & 12 \\
Distal & 9 \\
Percent diameter obstruction $(\%)$ & $48.66 \pm 17.82$ \\
\hline
\end{tabular}

In vivo

From the 24 patients selected for the study, two patients were excluded due to insufficient image quality for the $3 \mathrm{D}$ angiographic reconstruction and the subsequent analysis. The baseline characteristics for the remaining 22 patients are summarized in Table 1. A total of 78 reliable reference markers were identified from both angiographic and IVUS images. While the 22 most proximal markers were used as baseline positions for the distance mapping algorithm, the registration error was evaluated on the remaining 56 markers. The registration error ranges from -1.33 to $1.13 \mathrm{~mm}$, with an average value of $0.03 \pm 0.45 \mathrm{~mm}(P=0.67)$. A scatter plot of the registration error is presented by Fig. 4 . The error is not correlated to the distance between the evaluated marker and the baseline position $(P=0.73)$.

\section{Discussions}

The drug-eluting stents have proven to be able to reduce in-stent restenosis after coronary interventions [22-24]; however, the efficacy depends on the ability of the interventionalist to choose the optimal course of treatments and to implement the chosen course of action properly. Geographic mismatch due to suboptimal stent selection and positioning could significantly impact the short and long term outcome of the 


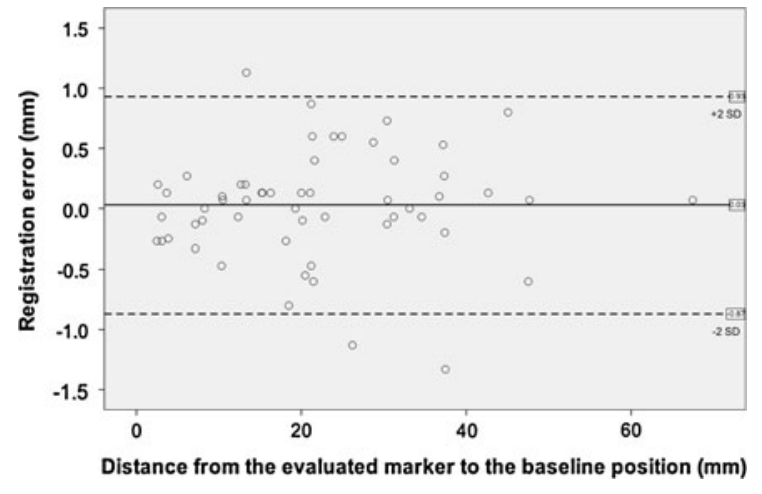

Fig. 4 The registration error for the XA-IVUS co-registration. The error is not correlated to the distance between the evaluated marker and the baseline position

stent-procedure [25, 26]. Sophisticated imaging and quantification tools are therefore demanded to guide the interventionalist to assess the true vessel dimensions, lesion location and extension for the optimization of the stent-procedure.

Quantitative coronary angiography was first developed to quantify vessel motion and the effects of pharmacological agents on the regression and progression of coronary artery disease [27]. It has developed substantially over the past decades and has been applied worldwide for research and clinical purposes, in both off-line and on-line situations [2]. Recently developed 3D systems based on routine angiographic projections have emerged as a new tool for the on-line guiding of coronary interventions. By resolving some of the well-known limitations in standard 2D analysis, e.g., vessel foreshortening and out-of-plane magnification [28], 3D QCA could provide more reliable assessments of segment length and diameter. In addition, the 3D angiographic reconstruction enables the subsequent automated determination of optimal viewing angles [8], which is considered to be clinically relevant in the stent deployment and positioning, especially for bifurcation related stent-procedures. Due to the overlap of coronary branches, the evaluation of bifurcation lesions from series of 2D projection images could be quite challenging. An example of the optimal bifurcation viewing angle is given by Fig. 5 . Figure $5 \mathrm{a}$ and $\mathrm{b}$ show the $2 \mathrm{D}$ angiographic projection and the $3 \mathrm{D}$ reconstructed bifurcation under $31 \mathrm{RAO}$, 33 Cranial, respectively. It is very clear from the 3D view that the visualization of the ostium of the diagonal branch is not optimal. Deploying a stent to the ostium of the diagonal branch by using this viewing angle could easily result in undesirable results, e.g., stent protrusion into the LAD or incomplete stent coverage at the ostium. Figure $5 \mathrm{c}$ shows the 3D bifurcation under the optimal viewing angle of 40 LAO, 56 Cranial. The visualization of the ostium of the diagonal branch has been significantly improved.

Thanks to the capacity and high resolution in imaging individual cross-sections of the coronary artery, IVUS and OCT have greatly improved our understanding of coronary atherosclerosis and the tissue responses after stent implantation. The role of IVUS/OCT in assessing plaque extent and distribution for optimal treatment planning has been well acknowledged. However, the ability in implementing the course of planning has been limited by the difficulty in corresponding IVUS/OCT with XA images. For stenting procedures, accurate positioning of the stent is important to ensure complete lesion

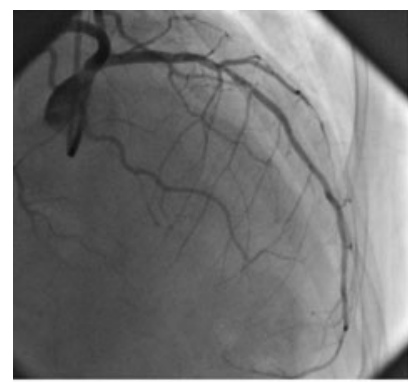

(a)

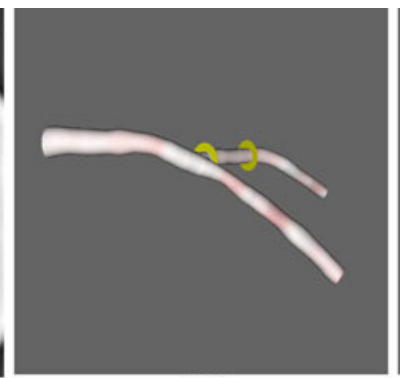

(b)

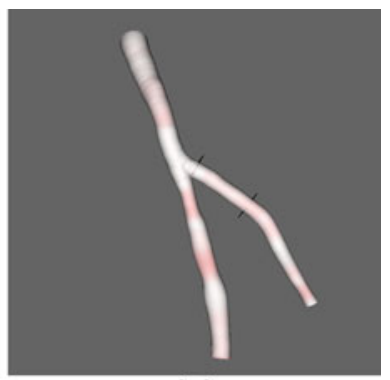

(c)
Fig. 5 The visualization of a bifurcation under different views: $\mathbf{a}$ is the angiographic view under 31 RAO, 33 Cranial; b is the $3 \mathrm{D}$ reconstructed bifurcation under 31 RAO, 33
Cranial; $\mathbf{c}$ is the 3D reconstructed bifurcation under the optimal viewing angle of $40 \mathrm{LAO}, 56$ Cranial 
coverage and to prevent the undesirable responds to the stent expansion, e.g., when stenting long diffused lesions with calcium deposited at the borders. The current workflow by mentally mapping the planned stenting position from IVUS/OCT to XA could be quite challenging when no landmark is available in the neighborhood of the lesion border. In other cases when the diseased vessel has multiple sidebranches, e.g., the LAD with many septal and diagonal branches, the mental mapping could be confused or even become completely mismatched due to the fact that not all sidebranches are well presented in the L-View of IVUS/OCT. In such cases, the XA-IVUS/ OCT co-registration could establish a point-to-point correspondence between XA and IVUS/OCT images. As a result, the deployment of the stent to the targeted position is simplified.

Despite of its attractive clinical perspectives, the integration of XA and IVUS/OCT in the current setting of catheterization laboratories has been hampered by the fact that the major angiography vendors do not have IVUS/OCT in their portfolio. The data connectivity is a significant bottleneck for such integration to be used in on-line mode. It is desirable that the angiography and IVUS/OCT vendors will cooperate to make the integration clinically acceptable with a seamless workflow in the near future.

The XA-IVUS/OCT co-registration serves the primary but yet the most important step for the complete fusion of 3D QCA and IVUS/OCT, which could be used to correct for the error in quantifying plaque volume introduced by vessel tortuosity [19]. In addition, in case of non-coaxial IVUS/OCT imaging $[29,30]$ when the transducer pullback trajectory is not aligned parallel to the vessel centerline, the fusion of these two imaging modalities can minimize the overestimation of cross-sectional dimensions from IVUS/OCT images, especially for the curved vessel segments with large diameter. However, such clinical benefits still have to be established.

\section{Limitations}

The IVUS/OCT pullback procedures were not ECG-gated, therefore, the cardiac motion could have influenced the axial position of each IVUS/OCT cross-sectional image. However, by interpreting the pullback series in a couple of longitudinal views, it is possible to visually identify the cross-sections that were significantly influenced by the cardiac motion and, to avoid using those positions as markers for the co-registration. Besides, the ECG-gated pullback procedures are not practical to be included in routine clinical practices for being expensive and for prolonging the acquisition time [31]. The faster pullback speed and higher frame rate acquisition achieved by the Fourier-domain OCT system could further decrease the impact of the motion-induced artifacts [29].

The in vivo validation is limited by the sample size and the usage of only one IVUS transducer pullback system. Early literatures have shown that the accuracy of in vivo length measurements using different pullback systems could be different [32], therefore, the co-registration could be impacted. Extensive validations for the co-registration by using different transducer pullback systems are under investigation.

\section{Conclusions}

The new XA-IVUS/OCT registration approach is a straightforward and reliable solution for the integration/combination of X-ray angiographic and IVUS/ OCT imaging. It provides the interventionalist with detailed information about vessel size and plaque size at every position along the vessel of interest, making this a suitable tool during the actual intervention.

Acknowledgments The authors thank Pieter Kitslaar, MSc (Medis), Jasper P. Janssen, MSc (LUMC), and Andrei Rares, $\mathrm{PhD}$ (LUMC) for their help in the computer programming, Joan C. Tuinenburg, MSc (LUMC \& Medis) for the discussions on QCA and Patrick Brouwer, MD (LUMC) for the help in the validation.

Conflict of interest Shengxian Tu and Gerhard Koning are employed by Medis medical imaging systems bv and have a research appointment at the Leiden University Medical Center. Johan H. C. Reiber is the CEO of Medis medical imaging systems bv, and has a part-time appointment at LUMC as Prof of Medical Imaging.

Open Access This article is distributed under the terms of the Creative Commons Attribution Noncommercial License which permits any noncommercial use, distribution, and reproduction in any medium, provided the original author(s) and source are credited. 


\section{References}

1. Reiber JHC, Serruys PW, Kooijman CJ et al (1985) Assessment of short-, medium-, and long-term variations in arterial dimensions from computer-assisted quantitation of coronary cineangiograms. Circulation 71:280-288

2. Reiber JHC, Tuinenburg JC, Koning G et al (2009) Chapter 2.2: quantitative coronary arteriography. In: Oudkerk M, Reiser MF (eds) Coronary radiology, medical radiology, 2nd revised edn, Springer, Berlin, pp 41-65

3. Goktekin O, Kaplan S, Dimopoulos K et al (2007) A new quantitative analysis system for the evaluation of coronary bifurcation lesions: comparison with current conventional methods. Catheter Cardiovasc Interv 69:172-180

4. Rittger H, Schertel B, Schmidt M et al (2009) Threedimensional reconstruction allows accurate quantification and length measurements of coronary artery stenoses. EuroIntervention 5:127-132

5. Tu S, Huang Z, Koning G et al (2010) A novel threedimensional quantitative coronary angiography system: in vivo comparison with intravascular ultrasound for assessing arterial segment length. Catheter Cardiovasc Interv 76: 291-298

6. Bruining N, Tanimoto S, Otsuka M et al (2008) Quantitative multi-modality imaging analysis of a bioabsorbable poly-L-lactid acid stent design in the acute phase: a comparison between 2 and 3D-QCA, QCU and QMSCT-CA. EuroIntervention 4:285-291

7. Schuurbiers JC, Lopez NG, Ligthart J et al (2009) In vivo validation of CAAS QCA-3D coronary reconstruction using fusion of angiography and intravascular ultrasound (ANGUS). Catheter Cardiovasc Interv 73:620-626

8. Tu S, Koning G, Jukema W et al (2010) Assessment of obstruction length and optimal viewing angle from biplane X-ray angiograms. Int J Cardiovasc Imaging 26:5-17

9. Green NE, Chen SJ, Hansgen AR et al (2005) Angiographic views used for percutaneous coronary interventions: a three dimensional analysis of physician-determined vs computer-generated views. Catheter Cardiovasc Interv 64:451-459

10. Sadamatsu K, Sagara S, Yamawaki T et al (2009) Threedimensional coronary imaging for the ostium of the left anterior descending artery. Int J Cardiovasc Imaging 25: 223-228

11. Gollapudi RR, Valencia R, Lee SS et al (2007) Utility of three-dimensional reconstruction of coronary angiography to guide percutaneous coronary intervention. Catheter Cardiovasc Interv 69:479-482

12. Glagov S, Weisenberg E, Zarins CK et al (1987) Compensatory enlargement of human atherosclerotic coronary arteries. N Engl J Med 316:1371-1375

13. Dumay ACM (1992) Image reconstruction from biplane angiographic projections. Dissertation, Delft University of Technology

14. Wahle A, Wellnhofer E, Mugaragu I et al (1995) Assessment of diffuse coronary artery disease by quantitative analysis of coronary morphology based upon 3-D reconstruction from biplane angiograms. IEEE Trans Med Imaging 14:230-241
15. Chen SJ, Carroll JD, Messenger JC (2002) Quantitative Analysis of Reconstructed 3-D Coronary Arterial Tree and Intracoronary Devices. IEEE Trans Med Imaging 21:724-740

16. Janssen JP, Koning G, De Koning PJH et al (2005) A new approach to contour detection in X-ray arteriograms: the wavecontour. Invest Radiol 40:514-520

17. Reiber JHC, van der Zwet PM, Koning G et al (1993) Accuracy and precision of quantitative digital coronary arteriography: observer-, short-, and medium-term variabilities. Cathet Cardiovasc Diagn 28:187-198

18. Slager CJ, Wentzel JJ, Schuurbiers JCH et al (2000) True 3-Dimensional reconstruction of coronary arteries in patients by fusion of angiography and IVUS (ANGUS) and its quantitative validation. Circulation 102:511-516

19. Wahle A, Lopez JJ, Olszewski ME et al (2006) Plaque development, vessel curvature, and wall shear stress in coronary arteries assessed by X-ray angiography and intravascular ultrasound. Med Image Anal 10:615-631

20. Rotger D, Radeva P, Canero C et al (2001) Corresponding IVUS and angiogram image data. Proc Comput Cardiol 28:273-276

21. Tu S, Koning G, Tuinenburg JC et al (2009) Coronary angiography enhancement for visualization. Int $\mathrm{J}$ Cardiovasc Imaging 25:657-667

22. Moses JW, Leon MB, Popma JJ et al (2003) Sirolimuseluting stents versus standard stents in patients with stenosis in a native coronary artery. N Engl J Med 349: $1315-1323$

23. Stone GW, Ellis SG, Cox DA et al (2004) A polymerbased, paclitaxel-eluting stent in patients with coronary artery disease. N Engl J Med 350:221-231

24. Stone GW, Moses JW, Ellis SG et al (2007) Safety and efficacy of sirolimus- and paclitaxel-eluting coronary stents. N Engl J Med 356:998-1008

25. Fujii K, Carlier SG, Mintz GS et al (2005) Stent underexpansion and residual reference segment stenosis are related to stent thrombosis after sirolimus-eluting stent implantation: an intravascular ultrasound study. J Am Coll Cardiol 45:995-998

26. Costa MA, Angiolillo DJ, Tannenbaum M et al (2008) Impact of stent deployment procedural factors on longterm effectiveness and safety of sirolimus-eluting stents (final results of the multicenter prospective STLLR trial). Am J Cardiol 101:1704-1711

27. Brown BG, Bolson E, Frimer M et al (1977) Quantitative coronary angiography: estimation of dimensions, hemodynamic resistance, and atheroma mass of coronary artery lesions using arteriography in 256 nonoperated patients. Circulation 55:329-337

28. Koning G, Hekking E, Kemppainen JS et al (2001) Suitability of the Cordis StabilizerTM marker guide wire for quantitative coronary angiography calibration: an in vitro and in vivo study. Catheter Cardiovasc Interv 52:334-341

29. Sawada T, Shite J, Negi N et al (2009) Factors that influence measurements and accurate evaluation of stent apposition by optical coherence tomography. Assessment using a phantom model. Circ J 73:1841-1847

30. Geselschap JH, Heilbron MJ, Hussain FM et al (1998) The effect of angulation on intravascular ultrasound imaging observed in vascular phantoms. J Endovasc Surg 5:126-133 
31. Bruining N, von Birgelen C, de Feyter PJ et al (1998) ECG-gated versus nongated three-dimensional intracoronary untrasound analysis: implications for volumetric measurements. Cathe Cardiovasc Diagn 43:254-260
32. Tanaka K, Carlier SG, Mintz GS et al (2007) The accuracy of length measurements using different intravascular ultrasound motorized transducer pullback systems. Int J Cardiovasc Imaging 23:733-738 